\title{
A Conserved Clathrin Assembly Motif Essential for Synaptic Vesicle Endocytosis
}

\author{
Jennifer R. Morgan, ${ }^{1,3}$ Kondury Prasad,2,3 Weihua Hao, ${ }^{2}$ George J. Augustine,, ${ }^{1,3}$ and Eileen M. Lafer ${ }^{2,3}$ \\ 1 Department of Neurobiology, Duke University Medical Center, Durham, North Carolina 27710, ${ }^{2}$ Department of \\ Biochemistry, University of Texas Health Science Center at San Antonio, San Antonio, Texas, 78229, and ${ }^{3}$ Marine \\ Biological Laboratory, Woods Hole, Massachusetts 02543
}

\begin{abstract}
Although clathrin assembly by adaptor proteins (APs) plays a major role in the recycling of synaptic vesicles, the molecular mechanism that allows APs to assemble clathrin is poorly understood. Here we demonstrate that AP180, like AP-2 and AP-3, binds to the $\mathrm{N}$-terminal domain of clathrin. Sequence analysis reveals a motif, containing the sequence DLL, that exists in multiple copies in many clathrin APs. Progressive deletion of these motifs caused a gradual reduction in the ability of AP180 to assemble clathrin in vitro. Peptides from AP180 or AP-2 containing this motif also competitively inhibited clathrin assembly by either protein. Microinjection of these peptides into squid giant presynaptic terminals reversibly blocked synaptic transmission and inhibited synaptic vesicle endocytosis by preventing coated
\end{abstract}

pit formation at the plasma membrane. These results indicate that the DLL motif confers clathrin assembly properties to AP180 and AP-2 and, perhaps, to other APs. We propose that APs promote clathrin assembly by cross-linking clathrin triskelia via multivalent interactions between repeated DLL motifs in the APs and complementary binding sites on the $\mathrm{N}$-terminal domain of clathrin. These results reveal the structural basis for clathrin assembly and provide novel insights into the molecular mechanism of clathrin-mediated synaptic vesicle endocytosis.

Key words: AP180; AP-2; clathrin adaptors; coated vesicles; membrane trafficking; presynaptic terminals; synaptic transmission
Clathrin is important for membrane trafficking in all eukaryotic cells (Goldstein et al., 1985; Pearse, 1988; Schmid, 1997). Clathrin assembles into cages that facilitate the budding of membranes into smaller vesicles. Both clathrin assembly and the connection of this cage to budding membranes are mediated by clathrin adaptor/ assembly proteins (APs; Keen et al., 1979; Kirchhausen, 1999). These clathrin APs exist in various cellular compartments and are separated into two structurally distinct classes: tetrameric and monomeric APs. Tetrameric APs have two large subunits $(\alpha / \gamma / \delta / \epsilon$ and $\beta$ ), a medium subunit $(\mu)$, and a small subunit $(\sigma)$ (Keen, 1987; Ahle et al., 1988; Matsui and Kirchhausen, 1990). AP-1 is involved in vesicle trafficking from the trans-Golgi (Robinson, 1987; Ahle et al., 1988). AP-2 mediates protein sorting and endocytosis from the plasma membrane of all cells, including neurons (Robinson, 1987; Ahle et al., 1988; Gonzalez-Gaitan and Jackle, 1997; Kirchhausen et al., 1997). AP-3 mediates synaptic vesicle formation from endosomes and protein transport to vacuoles (Cowles et al., 1997; Dell'Angelica et al., 1998; Faundez et al., 1998). No function has been assigned to AP-4, although its cellular localization suggests a role in trans-Golgi transport (Dell'Angelica et al., 1999). Monomeric APs include AP180 and clathrin assembly lymphoid myeloid leukemia protein (CALM; Ahle and Ungewickell, 1986; Kohtz and Puszkin, 1988; Murphy et al., 1991; Sousa et al., 1992; Zhou et al., 1992, 1993; Tebar et al., 1999). AP180 is a synapse-specific protein that is important for synaptic vesicle endocytosis (Zhang et al.,

\footnotetext{
Received April 15, 2000; revised Aug. 8, 2000; accepted Aug. 16, 2000.

This work was supported by National Institutes of Health Grant NS29051, a grant from the Muscular Dystrophy Association, and a Marine Biological Laboratory fellowship (to E.M.L.); by National Institutes of Health Grant NS21624 and a Human Frontier Science program grant (to G.J.A.); and by Ruth K. Broad Biomedical Research Foundation and National Research Service Award fellowships (to J.R.M.) We thank M. Hale and S. Miller for performing the electron microscopy and S. Jin for performing the biosensor analysis. We also thank L. Bonewald and S. Mouton of the Institutional Protein Core Facility at University of Texas Health Science Center at San Antonio for peptide synthesis and T. Kirchhausen and E. ter Haar for the clathrin terminal domain construct and thoughtful discussions.

Correspondence should be addressed to Dr. George J. Augustine, Department of Neurobiology; Box 3209, Duke University Medical Center; Durham, NC 27710. E-mail: georgea@neuro.duke.edu.

Copyright (C) 2000 Society for Neuroscience $0270-6474 / 00 / 208667-10 \$ 15.00 / 0$
}

1998; McMahon, 1999; Morgan et al., 1999; Nonet et al., 1999). CALM is a ubiquitously expressed AP180 homolog that also may be involved in the formation of clathrin-coated pits (Dreyling et al., 1996; Tebar et al., 1999).

What remains to be determined is how these structurally diverse APs promote clathrin assembly. Clathrin binding regions of the $\beta 2$ subunit of AP- 2 and of the $\beta 3$ A subunit of AP- 3 have been mapped to amino acids 616-663 (Shih et al., 1995) and 630-638 (Dell'Angelica et al., 1998), respectively. Alignment of these regions with other clathrin binding proteins suggested that the sequence $\mathrm{L}(\mathrm{L}, \mathrm{I})(\mathrm{D}, \mathrm{E}, \mathrm{N})(\mathrm{L}, \mathrm{F})(\mathrm{D}, \mathrm{E})$ defines the core of a clathrin binding site, referred to as the "clathrin box" (Dell'Angelica et al., 1998; ter Haar et al., 2000). Here we extend this work with the discovery that the large subunits of tetrameric APs and monomeric AP180 contain multiple copies of a similar clathrin binding element. The element is $\sim 23$ amino acids long and comprises both a central core motif of DLL (for AP-1, AP-2, and AP180) or SLL (for AP-3 and AP-4) and surrounding conserved residues. We provide several lines of evidence that DLL motifs confer clathrin assembly properties on APs. The clathrin assembly domain of AP180 containing 12 DLL motifs binds directly to the N-terminal domain of the clathrin heavy chain. Progressive deletion of the DLL motifs in AP180 quantitatively reduces the clathrin assembly activity according to the number of remaining motifs. Peptides containing the DLL motif competitively inhibit both AP180- and AP-2-mediated clathrin assembly in vitro and synaptic transmission and synaptic vesicle endocytosis in vivo. Because DLL motifs are necessary for clathrin assembly and are present in multiple copies in many APs, we propose that they allow APs to assemble clathrin coats by cross-linking adjacent clathrin triskelia.

\section{MATERIALS AND METHODS}

Expression and purification of recombinant proteins. The $\mathrm{N}$-terminal domain [TD; amino acids (aa) 1-494] of the rat clathrin heavy chain (construct from T. Kirchhausen, Harvard University, Cambridge, MA) was expressed recombinantly in Escherichia coli BL21 cells and purified as described (ter Haar et al., 1998). Constructs expressing full-length AP180 (mouse AP180, aa 1-901) and deletion constructs C58 (aa 305-901), C42 (aa 469-901), C38 (aa 511-901), C27 (aa 623-901), C22 (aa 681-901), and C16 (aa 739-901) were prepared as described previously (Hao et al., 1999). 
C51 (aa 371-901) and C49 (aa 392-901) were made by PCR with the following sense primers: 5'-AGCTTCGGTCGACTCTCTGGGGGTGCGACCGCC-3' (C51), 5'-AGCTTCGGTCGACTCGTTCCCTGTGAAGCACCG-3' (C49), and a common antisense primer, 5'-GCTGAAATGCGGCCGCTCTTACAAGAAATCCTT-3'. A SalI restriction site was designed in the sense primers, and a Not I site was designed in the antisense primer. In all of the reactions, plasmid pGEX3X-F1-20 (AS15-) was used as the template (Ye and Lafer, 1995a). The PCR products were subcloned into the pGEX4T-1 expression vector (Pharmacia, Piscataway, NJ) at the SalI/NotI site. The recombinant proteins were expressed in BL21 cells and purified under the same conditions as described previously for glutathione $S$-transferase (GST)-AP180 (Ye and Lafer, 1995a). GST was removed by cleavage with either Factor Xa or thrombin for the experiments displayed in Figure 3.

Surface plasmon resonance. Experiments were performed on a BIAcore 2000 Surface Plasmon Resonance instrument, using CM5 research grade chips (BIAcore, Piscataway, NJ). The experiments were performed at $25^{\circ} \mathrm{C}$ with HEPES-buffered saline [HBS; containing (in mM): 10 HEPES, 150 $\mathrm{NaCl}$, and 3 EDTA plus $0.005 \%$ surfactant $\mathrm{P} 20, \mathrm{pH} 7.0$ ] as running buffer at a flow rate of $5 \mu \mathrm{l} / \mathrm{min}$. The surfaces of research grade CM5 chips were activated by a $6 \mathrm{~min}$ injection of a solution containing $50 \% \mathrm{~N}$-ethyl- $\mathrm{N}^{\prime}$ (dimethylaminopropyl) carbodiimide and $50 \% \mathrm{~N}$-hydroxy-succinimide. GST-C58 (3.6 kRU, where $1 \mathrm{kRU}=1000$ response units $=$ a change of $\sim 1$ $\mathrm{ng} / \mathrm{mm}^{2}$ in surface protein concentration) was immobilized on a chip at $\mathrm{pH}$ 4.7, and GST (3.4 kRU) was immobilized at pH 5.0. After immobilization, each surface was blocked by a $6 \mathrm{~min}$ injection of $1 \mathrm{~m}$ ethanolamine at $\mathrm{pH}$ 8.5. TD was passed over the surfaces for $3 \mathrm{~min}$ at the indicated concentrations, followed by a 4 min injection of HBS. Then the surfaces were regenerated by a 3 min injection of $4 \mathrm{~m}$ guanidine-HCl.

Sequence analysis. The self-homology plot of AP180 in Figure $2 A$ was generated with PLALIGN (Huang and Miller, 1991). The multiple sequence alignment in Figure $2 B$ was generated with Megalign running under the Lasergene software package (DNAStar, Madison, WI), using the Clustal method (Thompson et al., 1994) and the PAM250 residue weight table. The alignments displayed in Figure $2 B$ included the following sequences, with the indicated accession numbers: mouse AP180 (M83985), rat $\beta 1$ adaptin (M77245), rat $\beta 2$ adaptin (M77246), mouse $\alpha \mathrm{A}$ adaptin (X14971), mouse $\alpha$ C adaptin (X14972), mouse $\gamma$ adaptin (X54424), human $\beta 3 \mathrm{~A}$ adaptin (U91931), human $\beta 3 \mathrm{~B}$ adaptin (U37673), human $\beta 4$ adaptin (AF092094), and human $\delta$ adaptin (U91930). Residues were grouped according to a structural similarity consensus that considers both the physical/chemical properties and the size/shape of the residues. Residue groupings of the consensus are: D/E, H/K/R, N/Q, W/Y, S/T, F/L/I/M and $\mathrm{A} / \mathrm{G} / \mathrm{V}, \mathrm{P}, \mathrm{C}$. Statistical analysis of the AP180/AP-1/AP-2 alignment revealed that the scored motifs displayed an average identity to the structural similarity consensus of $48 \%$ (range $30-70 \%$ ). Statistical analysis of the AP-3/AP-4 alignment revealed that the scored motifs displayed an average identity to the structural similarity consensus of $45 \%$ (range 35-57\%).

Purification of coat proteins and clathrin assembly assays. Clathrin, AP180, and AP-2 were purified from bovine brain as described previously (Prasad and Lippoldt, 1988; Hao et al., 1999). The assembly of bovine brain clathrin into coats was measured by ultracentrifugation in the presence of the indicated clathrin APs (Morgan et al., 1999). Briefly, each AP was combined with clathrin in $10 \mathrm{~mm}$ Tris- $\mathrm{HCl}, \mathrm{pH} \mathrm{8.5}$, and clathrin assembly was initiated by adding $0.1 \mathrm{vol}$ of $1 \mathrm{M}$ MES-NaOH, $\mathrm{pH}$ 6.7. The final conditions in the assembly reaction were $0.5 \mu \mathrm{M}$ clathrin, $0.1 \mathrm{M}$ MES-NaOH, $9 \mathrm{~mm}$ Tris- $\mathrm{HCl}, \mathrm{pH}$ 6.7, and the indicated concentrations of APs in a volume of $200 \mu \mathrm{l}$. The mixture was incubated on ice for $45 \mathrm{~min}$ and then was centrifuged at $400,000 \times g$ at $4^{\circ} \mathrm{C}$ for $6 \mathrm{~min}$. The upper $80 \%$ of the supernatant was removed and analyzed by SDS-PAGE and Coomassie blue staining. The percentage of clathrin that was assembled was determined by the relative depletion of clathrin from the supernatants of the centrifuged solutions before and after assembly. This was quantified by densitometry of Coomassie blue-stained gels (Molecular Dynamics Personal Densitometer SI, Sunnyvale, CA), using Image Quant software. In the absence of APs, $\sim 5 \%$ of the clathrin sedimented, and this background clathrin assembly was subtracted from all data. Inhibitory peptides were designed from mouse AP180 and rat AP-2 ( $\beta 2$; see Table 1 for amino acid sequences). The effects of these peptides were examined under the following conditions: $0.5 \mu \mathrm{M}$ clathrin, $1.0-1.2 \mu \mathrm{M}$ AP180 or 1.5-2.0 $\mu \mathrm{M}$ AP-2, 0.1 M MES-NaOH, $9 \mathrm{~mm}$ Tris-HCl, $\mathrm{pH}$ 6.7, and $0-1000 \mu \mathrm{M}$ peptide. The amount of clathrin that was assembled was determined as described above. Clathrin assembled in the presence of AP180 or AP-2, but without peptides, was in the range of $50-55 \%$. Inhibition by the peptides was calculated as the relative amount of assembly in the presence of the peptides as compared with the assembly in the absence of the peptides. Peptides were synthesized at the Institutional Protein Core Facility of the University of Texas Health Science Center (San Antonio, TX). The formation of clathrin coats and the inhibition of their formation by peptides was assessed by electron microscopy, as described previously (Ye and Lafer, 1995b). Assembled clathrin coat structures were applied to freshly glow-discharged Formvar carbon-coated grids and negatively stained with $1 \%$ uranyl acetate. The specimens were viewed in a JEOL 1200 EX Transmission electron microscope (Peabody, MA) at a magnification of $72,400 \times$.

Physiological methods. Electrical measurements were made on giant synapses in isolated stellate ganglia of the squid, Loligo pealei (Morgan et al., 1999). Ganglia were superfused with oxygenated physiological saline at $10-15^{\circ} \mathrm{C}$ containing (in $\mathrm{mm}$ ): $466 \mathrm{NaCl}, 54 \mathrm{MgCl}_{2}, 11 \mathrm{CaCl}_{2}, 10 \mathrm{KCl}, 3$ $\mathrm{NaHCO}_{3}$, and $10 \mathrm{HEPES}$, pH 7.2. Electrodes filled with $3 \mathrm{M} \mathrm{KCl}$ were inserted into the presynaptic axon and the postsynaptic axon for the purposes of stimulating synaptic transmission and recording transmitter release, respectively. Synaptic transmission was evoked by injecting a current pulse $(0.7-1.9 \mu \mathrm{A})$ every $30 \mathrm{sec}(0.03 \mathrm{~Hz})$ into the presynaptic axon to elicit single action potentials. Transmitter release was measured by recording the initial rate of rise of postsynaptic potentials. Data were acquired via an Axoclamp-2A amplifier and analyzed with Axobasic programs written by Dr. Felix E. Schweizer (UCLA, Los Angeles, CA).

For microinjection the peptides were dissolved in physiological squid saline containing (in $\mathrm{mM}$ ): $250 \mathrm{~K}$-isothionate, $100 \mathrm{KCl}, 100$ taurine, and 50 HEPES, pH 7.4. These solutions were delivered from a microinjection pipette by delivering pulses of positive pressure (10-80 msec; $10-100 \mathrm{psi}$; $\mathrm{N}_{2}$ gas) from a Picospritzer injector (General Valve, $\mathrm{NJ}$ ). Peptides were coinjected with FITC-dextran (3 kDa molecular weight; $100 \mu \mathrm{M}$; Molecular Probes, Eugene, OR) to monitor the intraterminal peptide concentration. Fluorescence was imaged with a Zeiss Axioskop $(10 \times, 0.25$ numerical aperture objective; Oberkochen, Germany) and detected with a Cohu SIT camera attached to an image processor (Image-1, Universal Imaging, West Chester, PA).

To correlate the actions of AP2 peptides and mutants on clathrin assembly and synaptic transmission (see Fig. 7D), we chose a standard concentration at which to compare both the inhibition of clathrin assembly in vitro and the inhibition of synaptic transmission in vivo by each peptide. For clathrin assembly by AP-2, the standard concentration chosen was 0.3 $\mathrm{mm}$, the $\mathrm{IC}_{50}$ of AP2 pep for AP-2-mediated assembly (see Table 1). For clathrin assembly by AP180, the standard concentration chosen was 0.4 $\mathrm{mM}$, the $\mathrm{IC}_{50}$ of AP2 peptide on AP180-mediated clathrin assembly. The standard concentration for examining the effect of AP2 peptide and mutants on synaptic transmission was $2.3 \mathrm{~mm}$, the $\mathrm{IC}_{50}$ for inhibition of synaptic transmission by AP2 pep (see Fig. $7 C$ ). The mean inhibitory effects of each peptide in the in vitro and in vivo assays were measured at the standard concentrations and plotted as shown in Figure $7 D$.

Electron microscopy. Terminals were fixed with $2.5 \%$ glutaraldehyde and processed for electron microscopy as described in Sanchez et al. (1990). An $80-\mathrm{nm}$-thick section was taken every $50 \mu \mathrm{m}$ through the entire length of the synaptic terminal. Each section was magnified 12,000× and examined with a JEM-1200 ExII (JEOL) electron microscope. All sections from peptideinjected and control terminals were analyzed with Image-1 software (Universal Imaging).

Distances between the plasma membrane and centers of individual synaptic vesicles or coated vesicles were measured as described previously (Hess et al., 1993). Because active zones typically are spaced $\sim 1 \mu \mathrm{m}$ apart, we measured vesicles within $500 \mathrm{~nm}$ of the active zone to ensure that each vesicle was counted in association with only one active zone. The plasma membrane perimeter of each terminal was measured from lowmagnification $(500 \times)$ micrographs (Burns et al., 1998). Such measurements underestimate plasma membrane area because of small invaginations (Morgan et al., 1999). To determine the magnitude of this effect, we measured the perimeter of small regions of the plasma membrane in high-magnification images $(12,000 \times)$ and used it to correct perimeter measurements made at low magnification. Plasma membrane perimeter was multiplied by the thickness of the section $(80 \mathrm{~nm})$ to calculate the surface area of plasma membrane in each section. The surface area of synaptic and coated vesicles was calculated as $4 \pi r^{2}$, where $r$ is the measured radius of these vesicles. Surface area per vesicle was multiplied by the mean number of vesicles per active zone, yielding the amount of vesicle area per active zone, and multiplied by the number of active zones per section to yield the mean surface area in synaptic and coated vesicles in each section. Vesicular structures that were 100-200 nm in diameter were counted as vesicular endosomes (Burns et al., 1998). Because their diameters were larger than the thickness of the section, their surface area was calculated as $2 \pi r \theta$, where $\theta$ is the thickness of the section $(80 \mathrm{~nm})$.

\section{RESULTS}

\section{AP180 binds to the $\mathrm{N}$-terminal domain of clathrin heavy chain}

Recent work has demonstrated that both an AP-2 $\beta 2$ peptide and an AP-3 $\beta 3$ A peptide interact directly with the N-terminal domain (TD) of clathrin heavy chain (ter Haar et al., 2000). However, the identification of a binding site for AP180 on clathrin has been problematic. Previous studies revealed that "clipped cages" from which the TD was removed by proteolysis lost the ability to bind to AP180, suggesting that AP180 may bind to the TD. However, this may not be the case because the proteolytically released TD did not bind measurably to AP180 (Murphy and Keen, 1992). We used recombinant $\mathrm{TD}$ and sensitive surface plasmon resonance (SPR) detection to evaluate the binding interaction between AP180 and the TD of clathrin. The clathrin assembly domain of AP180 (C58, aa 305-901), fused to GST, was immobilized on an SPR chip. As a 
A

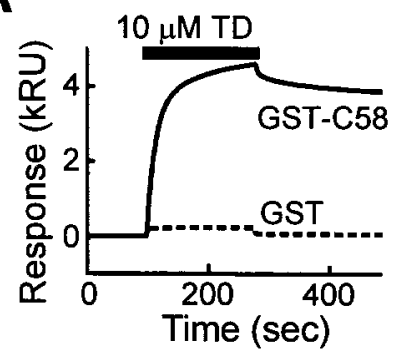

B

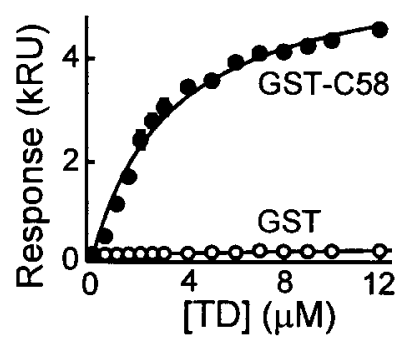

Figure 1. Binding of clathrin TD to AP180. A, Time course of binding of TD to AP180 was monitored by SPR. Traces illustrate the SPR response, expressed in kilo response units $(k R U)$. During the time indicated by the bar, TD was passed over surfaces to which either GST-C58 (solid line) or GST (dashed line) had been coupled covalently. B, TD binds to GST-C58 in a dose-dependent manner, whereas TD does not bind to GST at all of the concentrations that were tested. Different concentrations of TD were passed over the surfaces, and the responses were followed over time. Maximum response values are plotted as a function of TD concentration. The data points represent the mean response in two experiments. Error bars indicate \pm SEM. The data were fit by a rectangular hyperbolic function.

control, GST was immobilized on a second surface. Purified recombinant TD was passed over the two surfaces, and the refractive index at the surfaces was monitored continuously. The binding of TD to GST-C58 is expected to change the surface refractive index in proportion to the mass of TD that is bound. When TD was passed over the GST-C58 surface, a time-dependent increase of the response was observed, indicating that TD was binding to GST-C58 (Fig. 1A). When the TD solution was replaced by protein-free buffer, a slow dissociation of the bound TD was observed. This is in contrast to the small SPR signal observed while TD passed over the GST surface, presumably attributable to changes in solution flow (Fig. 1 $A$ ). Furthermore, SPR analysis indicated that TD specifically bound to C58 in a dose-dependent manner (Fig. 1B). Similar results were obtained when full-length bovine AP180 was used instead of the C58 domain (data not shown). TD did not interact with control surfaces prepared with BSA and $\mathrm{IgG}$, and BSA and IgG also did not interact with the specific GST-C58 surface. These results indicate that AP180, like AP-2 (Murphy and Keen, 1992; ter Haar et al., 2000) and AP-3 (Dell'Angelica et al., 1998; ter Haar et al., 2000), specifically binds to TD.

\section{A repeated clathrin assembly motif in many APs}

We next wanted to identify the site on AP180 to which clathrin binds. The entire C-terminal domain of AP180 is required for efficient assembly of clathrin (Ye and Lafer, 1995a), suggesting that clathrin assembly activity is distributed throughout this domain. Further, self-homology plots reveal that this domain of mammalian AP180 consists of a large number of repeated sequence elements (Zhou et al., 1993) (Fig. 2A). To consider whether the repeating structures might represent clathrin binding sites, we made an internal alignment of the primary amino acid sequence of AP180, guided by the use of a structural similarity consensus. This alignment revealed that the clathrin assembly domain of AP180 contains 12 repeats of a degenerate sequence, $\sim 23$ amino acids in length, that frequently contains a central DLL motif (Fig. 2B). Very similar repeating motifs also are found in CALM, as well as in squid, Drosophila, and Caenorhabditis elegans AP180 (data not shown). The clathrin assembly $(\beta 2)$ subunit of AP-2 possesses three similar repeats (Fig. $2 B$ ), two of which map to the clathrin binding region previously identified between amino acids 616-663 (Shih et al., 1995). Multiple repeats also were found in the corresponding regions of the AP-1 $\beta 1$ subunit and in the other large subunits of AP-1 $(\gamma)$ and AP-2 ( $\alpha \mathrm{A}$ and $\alpha \mathrm{C}$; Fig. $2 B)$. The $\alpha$ subunits of AP-2 have been reported to bind clathrin, although it is not known whether they can assemble clathrin (Prasad and Keen, 1991; Goodman and Keen, 1995). The large subunits of AP-3 ( $\delta$; $\beta 3 \mathrm{~A}$, and $\beta 3 \mathrm{~B})$ and AP-4 $(\beta 4)$ contain similar repeats, typically centered on an SLL sequence (Fig. $2 B$ ). Previous mutagenesis of

A

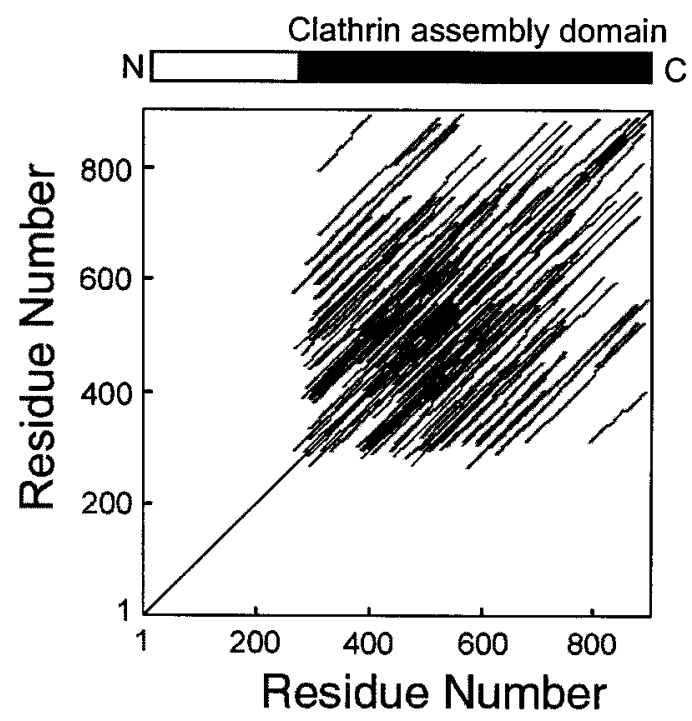

B
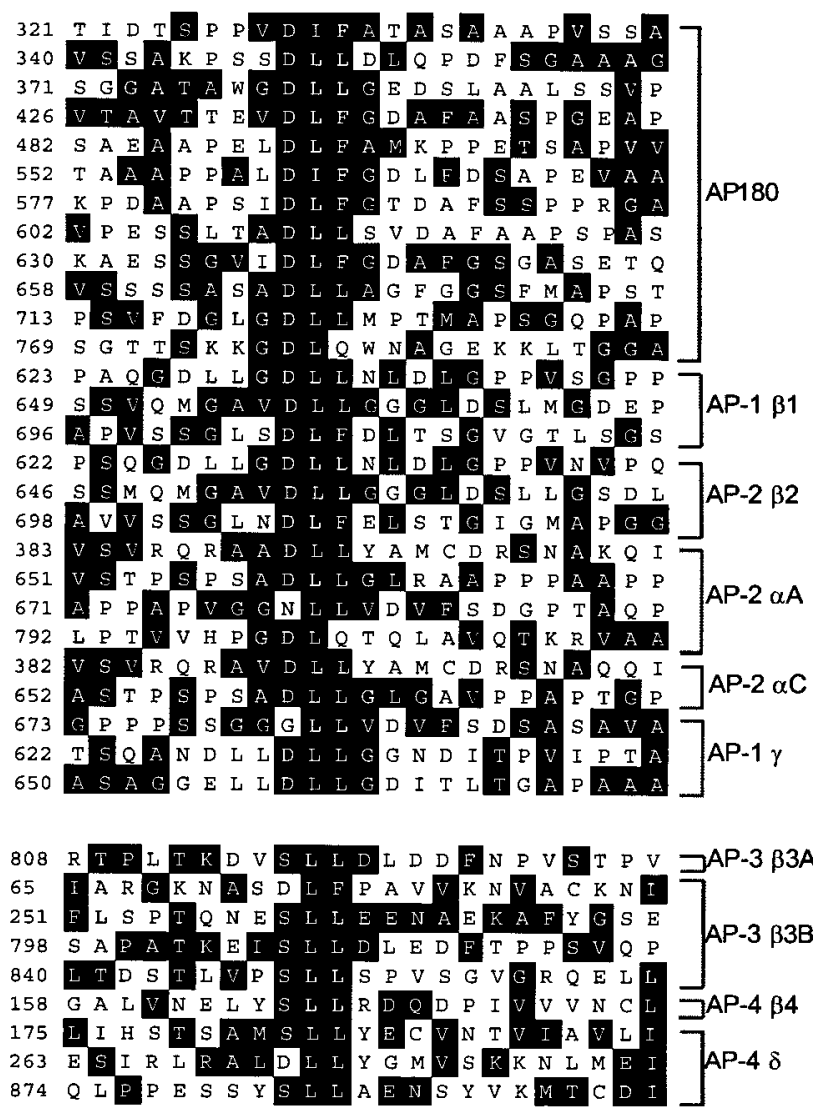

Figure 2. Identification of a repetitive element in the APs. $A$, A PLALIGN self-homology plot of AP180 reveals that the structure of the clathrin assembly domain of AP180 (residues 305-901) is highly repetitive. The parallel lines indicate regions of similarity. $B$, Inspection of the LALIGN alignments revealed that the repetitive element is an $\sim 23$ amino acid degenerate sequence, typically included on a core DLL motif. The AP180 repeats were aligned with similar repeats found in the large subunits of the AP-1 and AP-2 adaptors by Megalign. Below, Alignment of the large subunits of the AP-3 and AP-4 adaptors revealed a related repetitive element that typically included a core SLL motif. The residues surrounded by black in the alignments are identical to a structural similarity consensus (see Materials and Methods). 
A

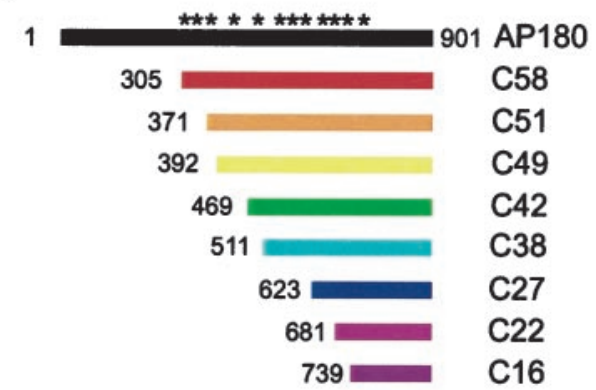

B

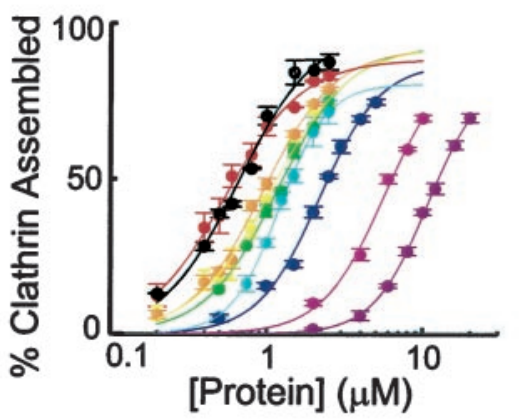

C

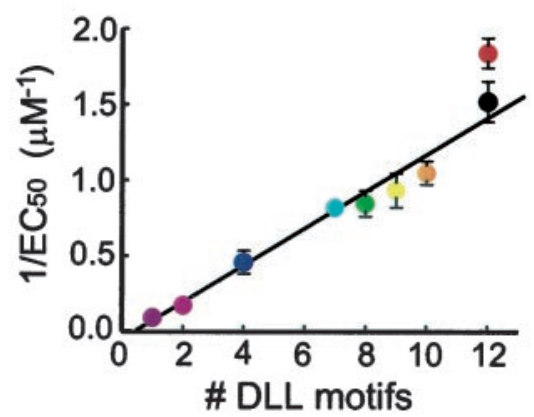

D

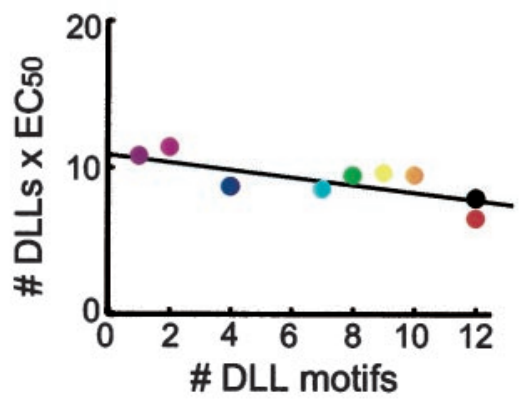

Figure 3. Deletion of DLL motifs reduces the ability of AP180 to assemble clathrin. $A$, Recombinant deletion mutants of AP180 were constructed to vary the number of DLL motifs in the clathrin assembly domain (C58). The DLL motifs within full-length AP180 are denoted by asterisks. B, Deletion of the DLL motifs gradually reduced the ability of the AP180 mutants to assemble clathrin in vitro. Colors correspond to the constructs indicated in $A$ for $B-D$; the data points represent the mean of three to six independent experiments; error bars indicate \pm SEM. Smooth lines indicate the Hill equation fit to the data. $C$, The clathrin assembly activity of the AP180 mutants was a linear function of the number of DLL motifs. The concentration of assembly protein to give half-maximal assembly $\left(E C_{50}\right)$ was determined from fitting the data in $B$ to the Hill equation. The error bars indicate \pm SEM. $D$, Clathrin assembly is proportional to the concentration of DLL motifs, because the product of the $\mathrm{EC}_{50} \times$ the number of DLLs motifs was constant for each construct. The $\mathrm{EC}_{50}$ values were determined from the data in $B$.

$\beta 3 \mathrm{~A}$ revealed that the sequence SLLDLDDFN, contained within one of these repeats (Fig. $2 B$ ), constitutes a clathrin binding site (Dell'Angelica et al., 1998). Thus, our analysis identified a repeated motif centered on a DLL/SLL that is common to many APs and

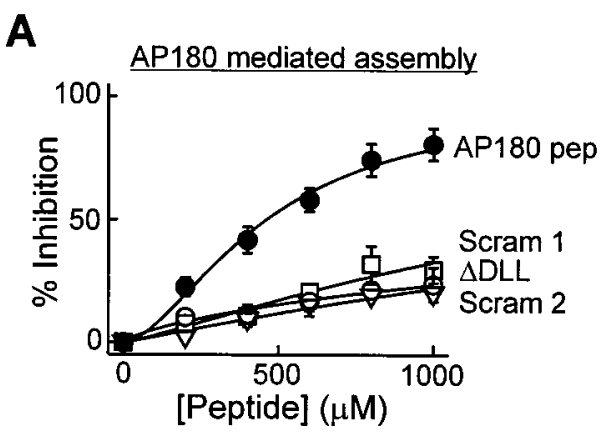

B

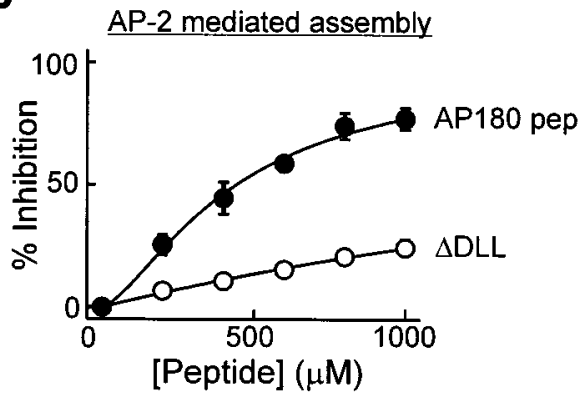

Figure 4. A DLL-containing peptide from the clathrin assembly domain of AP180 (AP180 pep) inhibits clathrin assembly in vitro by both AP180 and AP-2. $A$, AP180 pep produced a concentration-dependent inhibition of clathrin assembly by AP180. The inhibition by AP180 pep was reduced when the DLL was mutated to AAA $(\Delta D L L)$ or when the sequence of amino acids within the peptide was scrambled (Scram1 and Scram2). B, Concentration-dependent inhibition of AP-2-mediated clathrin assembly by AP180 pep. The inhibition by AP180 pep was reduced when the DLL was mutated to AAA $(\triangle D L L)$. In both panels the data points represent the mean of three to four independent experiments; error bars indicate \pm SEM. Smooth lines are fits of the Hill equation.

might confer clathrin binding and assembly properties on these proteins.

To determine whether the repeated motifs are important for clathrin assembly, we first constructed AP180 mutants with progressive deletions throughout the $\mathrm{C}$-terminal clathrin assembly domain (Fig. 3A). Progressive deletion of the clathrin assembly domain of AP180 gradually decreased the number of DLL motifs and caused a parallel loss in the clathrin assembly activity of AP180 (Fig. $3 B$ ). For the nine constructs that we studied, there was a linear relationship ( $r=0.96)$ between the number of DLL motifs and the clathrin assembly activity, indicated by the $\mathrm{EC}_{50}$ (Fig. $3 C$ ). We found that clathrin assembly is dependent on the concentration of DLL motifs; when the $\mathrm{EC}_{50}$ for each construct was normalized for the number of DLLs, by multiplying the $\mathrm{EC}_{50}$ times the number of DLLs, the product was remarkably constant $($ Fig. $3 D$; mean $=$ $9.1 \pm 0.5 \mu \mathrm{M})$. This indicates that clathrin assembly is directly proportional to the concentration of DLL motifs and thereby implicates these repeated motifs in clathrin assembly.

\section{DLL motif peptide from AP180 inhibits clathrin assembly and synaptic transmission}

If the DLL motifs are important for clathrin binding and assembly, then peptides bearing these motifs should act as competitive blockers of AP-induced clathrin assembly. Clathrin assembly was studied as a stringent measure of biological activity, because some proteins can bind clathrin without assembling it (Ye and Lafer, 1995b; Goodman et al., 1997). A DLL-containing peptide from AP180 (AP180 pep; aa 371-391) was synthesized and tested in vitro for its ability to inhibit clathrin assembly by APs. Mammalian APs were used in this analysis, because squid AP-2 has not been purified or cloned. AP180 pep inhibited AP180-mediated clathrin assembly in a dose-dependent manner (Fig. 4A). The relationship between peptide concentration and the degree of inhibition of clathrin assembly could be described by the Hill equation (smooth 
Table 1. Inhibition of clathrin assembly and synaptic transmission by AP180 and AP-2 peptides

\begin{tabular}{|c|c|c|c|c|}
\hline AP180 peptides & Sequence & $\begin{array}{l}\mathrm{IC}_{50} \text { for } \mathrm{AP}-2- \\
\text { mediated assembly }\end{array}$ & $\begin{array}{l}\mathrm{IC}_{50} \text { for AP180- } \\
\text { mediated assembly }\end{array}$ & $\begin{array}{l}\text { Mean inhibition } \\
\text { of transmission }\end{array}$ \\
\hline AP180 pep & SGGATAWGDLLGEDSLAALSS & $0.4 \mathrm{mM}$ & $0.5 \mathrm{mM}$ & $57 \% \pm 5.8(20)^{a}$ \\
\hline AP180 pep $\Delta \mathrm{DLL}$ & SGGATAWGAAAGEDSLAALSS & $3.1^{b}$ & $4.9^{b}$ & $0(2)$ \\
\hline Scram AP180 pep 1 & SGLLDSADAEGLTAAWGLGSS & $\mathrm{ND}^{c}$ & $1.8^{b}$ & $6.6 \pm 2.8(5)$ \\
\hline Scram AP180 pep 2 & GSSLGLASEAADGSDWLLATG & ND & $3.3^{b}$ & ND \\
\hline
\end{tabular}

AP-2 peptides

\begin{tabular}{|c|c|c|c|c|}
\hline AP2 pep & QGDLLGDLLNLDLGP PVNVPQ & 0.3 & 0.4 & $43 \pm 6.0(28)$ \\
\hline AP2 pep $\Delta$ PVN & QGDLLGDLLNLDLGP $\underline{A A A V P Q}$ & 0.2 & 0.4 & $49 \pm 14(4)$ \\
\hline AP2 pep $\Delta$ NLD & QGDLLGDLLAAALGPPVNVPQ & 0.7 & 0.9 & $43 \pm 11(4)$ \\
\hline AP2 pep $\Delta$ DLL & QGDLLGAAANLDLGPPVNVPQ & $28^{b}$ & $2.5^{b}$ & $4.0 \pm 2.6(7)$ \\
\hline AP2 pep D/L swap & QGLDDGLDDNDLDGP PVNVPQ & $14^{b}$ & $50^{b}$ & $0(3)$ \\
\hline
\end{tabular}

${ }^{a}$ Numbers in parentheses indicate the number of experiments performed.

${ }^{b}$ Because inhibition was $<50 \%$ at $1 \mathrm{~mm}$, these values are extrapolations.

${ }^{c} \mathrm{ND}$, Not determined.

lines in Fig. 4A); the concentration of peptide required for halfmaximal inhibition of assembly $\left(\mathrm{IC}_{50}\right), 0.5 \mathrm{~mm}$ in this case, is reported in Table 1 . This inhibitory activity depended on the presence of the DLL motif, because scrambled AP180 peptides (Scram AP180 pep 1 and 2) and a peptide in which the DLL was mutated to AAA (AP180 pep $\triangle \mathrm{DLL}$ ) were poor inhibitors of clathrin assembly (Fig. 4A). Compared with AP180 pep, these mutant peptides were fourfold to 10 -fold weaker inhibitors (see Table 1). AP180 pep also inhibited assembly of clathrin by squid AP180, whereas the mutated and scrambled peptides did not (data not shown). If the DLL motifs exist within a clathrin binding site common to many clathrin APs, then AP180 pep should crossinhibit the assembly of clathrin that is mediated by other APs. We found that AP180 pep indeed could inhibit AP-2-mediated clathrin assembly in vitro, whereas AP180 pep $\triangle \mathrm{DLL}$ was eight times weaker (Fig. 4B, Table 1). These results indicate that the DLL motif defines a clathrin assembly motif for AP180 and AP-2 and suggest that this conclusion may be applicable to other APs.

Given its actions in vitro, AP180 pep should act as a broad spectrum inhibitor of clathrin assembly in vivo, and this was examined by injecting this peptide into squid giant presynaptic terminals. As a first test, synaptic transmission was measured by monitoring the postsynaptic response to presynaptic action potentials elicited at a low frequency $(0.03 \mathrm{~Hz})$. If AP180 pep inhibits clathrin assembly, then synaptic transmission should be inhibited as synaptic vesicles continue to fuse with the plasma membrane but are not retrieved and restored to the pool of releasable vesicles (Morgan et al., 1999). AP180 pep inhibited synaptic transmission without affecting the presynaptic action potential (Fig. $5 A$ ). Because AP180 pep was injected into only the presynaptic terminal, this peptide works by inhibiting transmitter release rather than by affecting the postsynaptic neuron. This inhibition was reversible and therefore was not attributable to microinjection damage (Fig. 5B, Table 1). AP180 pep $\Delta$ DLL, which had very little effect on clathrin assembly in vitro, had no effect on synaptic transmission when it was injected into the presynaptic terminal (Fig. 5C). Thus, the effects of these peptides on synaptic transmission paralleled their actions on clathrin assembly in vitro, suggesting that the inhibition of transmission is attributable to the blockade of clathrin assembly.

\section{DLL motif peptide from AP-2 also inhibits clathrin assembly and synaptic transmission}

As another test of the role of DLL motifs in clathrin assembly, we examined a DLL-containing peptide from the $\beta 2$ subunit of AP-2 (AP2 pep; aa 624-644). Like AP180 pep, in vitro AP2 pep inhibited the assembly of clathrin by either AP-2 (Fig. $6 A$, Table 1 ) or AP180 (Fig. $6 B$, Table 1). Replacement of the DLL by AAA (AP2 pep $\triangle \mathrm{DLL})$ greatly reduced the inhibitory effect of this peptide on clathrin assembly (Fig. 6A,B). To examine the structural basis for this effect, we examined clathrin cages made with purified AP180 via electron microscopy. Clathrin cages were formed in the presence of AP2 pep $\triangle$ DLL (Fig. $6 C$ ), but not in the presence of AP2 pep (Fig. 6D). AP2 pep also reversibly inhibited synaptic transmission when it was injected into the squid giant presynaptic terminal (Fig. 7A, Table 1). This inhibition was dose-dependent, with the peptide capable of completely inhibiting synaptic transmission at maximal concentrations (Fig. $7 C$ ). Inhibition of synaptic transmission occurred at peptide concentrations somewhat higher than those needed to block clathrin assembly in vitro; this may reflect differences in the affinity of the peptides for squid clathrin (vs mammalian clathrin) or in the amount of APs and clathrin that are present in vivo (vs in vitro). In contrast, AP2 pep $\Delta \mathrm{DLL}$ had no effect on synaptic transmission at all of the concentrations that were tested (Fig. $7 B, C$ ). Thus, DLL motifs within AP-2 are also important for clathrin assembly and synaptic transmission.

To define further the sequence requirements for clathrin assembly and peptide action, we prepared additional peptides that included mutations both within the DLL sequence and elsewhere in AP2 pep. When the DLL was replaced with AAA (AP2 pep $\triangle \mathrm{DLL}$ ) or when the $\mathrm{D}$ and $\mathrm{L}$ residues were exchanged (AP2 pep $\mathrm{D} / \mathrm{L}$ swap), the ability to inhibit clathrin assembly was reduced sixto 125 -fold (Fig. $6 A, B$, Table 1 ). In contrast, mutation of the adjacent NLD residues (AP2 pep $\triangle$ NLD) reduced the inhibitory activity of the peptide only twofold, and mutation of the PVN sequence further downstream (AP2 pep $\triangle \mathrm{PVN}$ ) had no effect (Fig. $6 A, B$, Table 1). Each of these mutated AP-2 peptides also was tested for its ability to inhibit synaptic transmission. The actions of these peptides in vivo were very similar to their actions in vitro; AP-2 peptides containing DLLs were effective in inhibiting synaptic transmission, whereas those with mutated DLLs had no effect (see Table 1). There was a strong correlation $(r=0.98)$ between the inhibitory actions of these peptides on clathrin assembly and synaptic transmission (Fig. 7D). Identical results were seen when the inhibition of clathrin assembly by AP180 was compared with the inhibition of transmission by AP2 pep and mutants (data not shown). This indicates that the DLL is essential for the activity of AP2 pep and that the NLD makes a smaller contribution to the activity both in vitro and in vivo. All of these conclusions support the hypothesis that the DLL motif is an important determinant of clathrin binding and assembly by APs.

\section{DLL motif peptide inhibits endocytosis at the clathrin assembly step}

The ability of the AP peptides to inhibit synaptic transmission is consistent with these peptides blocking clathrin-mediated endocytosis in the nerve terminal. We next examined this possibility more 


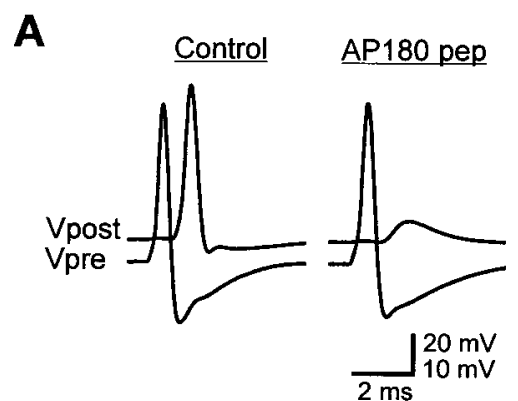

B

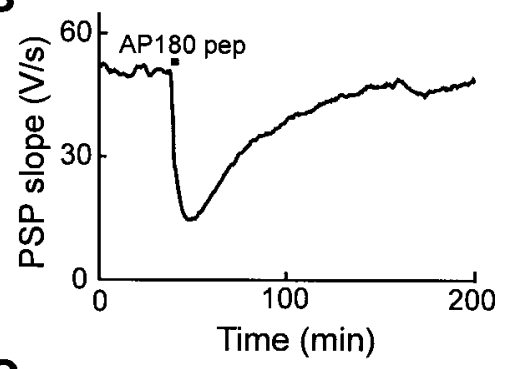

C

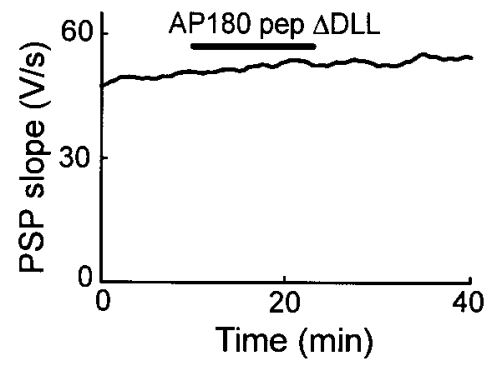

Figure 5. AP180 pep inhibits neurotransmitter release. $A$, Recordings of presynaptic and postsynaptic responses before (Control) and after the injection of AP180 pep. AP180 pep transiently reduced transmitter release below the threshold for eliciting a postsynaptic action potential. The vertical scale bar applies to Vpost (above) and Vpre (below) traces. B. Time course of the inhibition of transmitter release by AP180 pep, (injected during the time indicated by the bar), as measured by the slope of the postsynaptic potential (PSP). $C$, AP180 pep $\triangle \mathrm{DLL}$ had no effect on transmitter release.

directly by examining the ultrastructure of squid presynaptic terminals that were injected with AP2 pep. If AP2 pep inhibits clathrin assembly in vivo, then this peptide should deplete the nerve terminal of both synaptic vesicles and coated vesicles. Further, the accumulation of vesicular membrane should cause a proportionate increase in the area of the presynaptic plasma membrane (Heuser and Reese, 1973; Morgan et al., 1999). To test these predictions, we injected AP2 pep until synaptic transmission was inhibited by $>90 \%$. This was followed by fixation in glutaraldehyde and subsequent analysis by electron microscopy. As a control, similar concentrations of an inert peptide, AP2 pep $\triangle \mathrm{DLL}$, were injected into other terminals and had no effect on synaptic transmission, as described above.

In comparison to controls, terminals injected with AP2 pep were depleted of synaptic vesicles and coated vesicles (Fig. 8). These structural changes were analyzed in a number of ways. We quantified the spatial distribution of synaptic vesicles, by measuring the distance between these vesicles and the active zones at the plasma membrane (Hess et al., 1993), and found that AP2 pep caused a depletion of synaptic vesicles at all distances (Fig. 9A). When normalized to the distribution of vesicles in the control terminals, the AP2 peptide caused a $23 \%$ reduction in the docked synaptic vesicles within $50 \mathrm{~nm}$ of the active zone and a $66 \%$ depletion of vesicles farther away (Fig. $9 B$ ). This yielded a $62 \%$ overall loss of synaptic vesicles in the AP2 peptide-injected terminals (Fig. 9C; $p<0.01$, Student's $t$ test). We also measured the number of coated vesicles and found that these were depleted by $95 \%$, even more
A

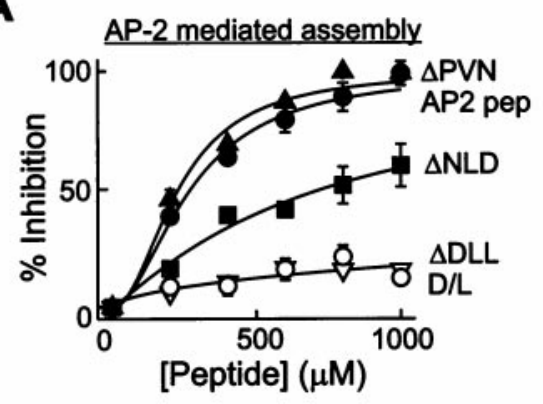

B

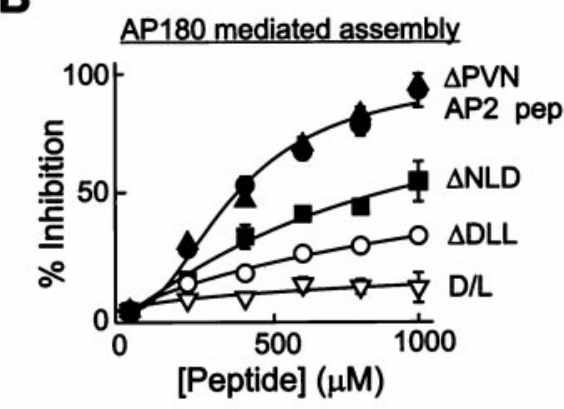

C

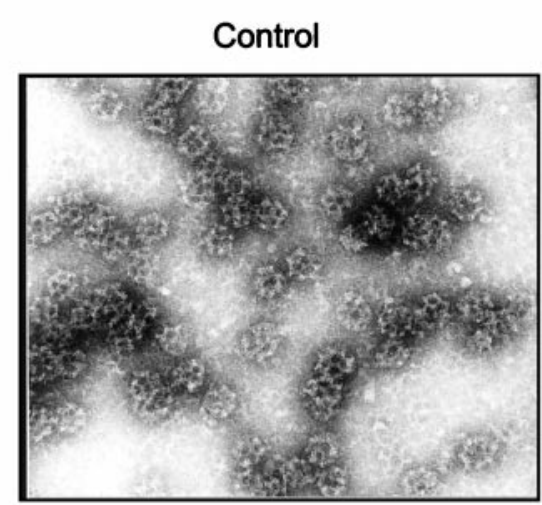

D

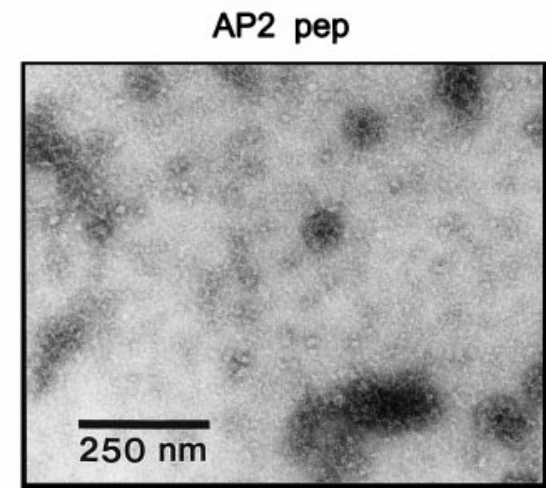

Figure 6. A DLL-containing AP-2 peptide ( $A P 2 \mathrm{pep})$ inhibits clathrin assembly in vitro. $A, B$, Clathrin assembly by both AP-2 $(A)$ and AP180 $(B)$ was inhibited by AP2 pep in a concentration-dependent manner, whereas a peptide in which the DLL was mutated to AAA $(\triangle D L L)$ or in which the D and $L$ residues were exchanged $(D / L)$ had little effect. Mutation of the downstream PVN to AAA in AP2 pep $(\triangle P V N)$ did not reduce the efficacy of the peptide, whereas the mutation of the adjacent NLD to AAA $(\triangle N L D)$ had a moderate effect. Data points represent the mean of three to four independent experiments; error bars indicate \pm SEM. Smooth lines are fits of the Hill equation. $C, D$, The ability of AP2 pep to inhibit clathrin assembly also was examined by negative-staining electron microscopy of clathrin cages that were assembled in the presence of AP2 pep $\triangle \mathrm{DLL}$ (Control) or AP2 peptide (AP2 pep). The scale bar in $D$ applies to both images. 
A

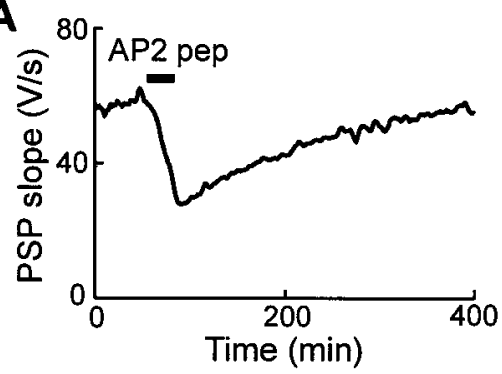

B

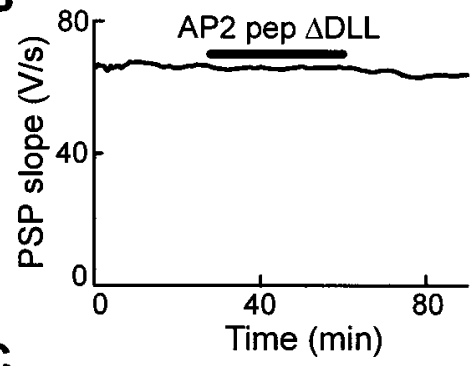

C

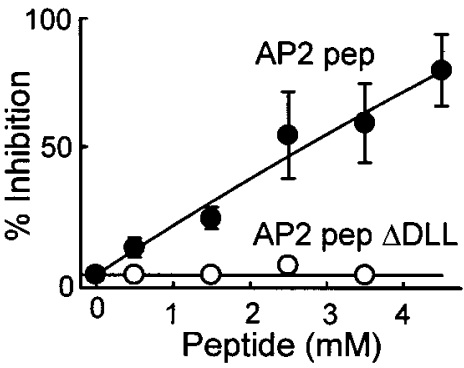

D

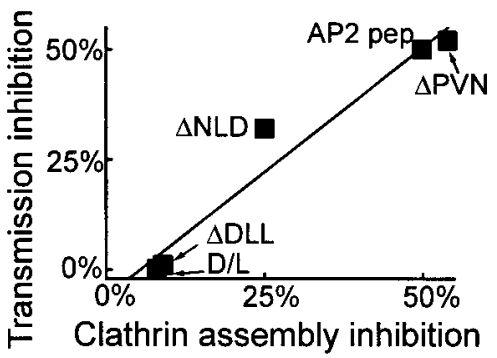

Figure 7. AP2 pep also inhibits transmitter release. $A$, AP2 pep reversibly inhibited transmitter release when it was injected (bar) into the squid giant presynaptic terminal. $B$, The inhibitory effect on transmission was lost when the DLL was mutated $(A P 2$ pep $\triangle D L L)$. $C$, Inhibition of transmitter release by AP2 pep was dependent on peptide concentration. Data points represent the mean inhibition measured in two to seven experiments; error bars represent \pm SEM. Smooth lines indicate rectangular hyperbola fits to the data. $D$, Correlation between the abilities of several AP-2 peptides to inhibit synaptic transmission in vivo and clathrin assembly in vitro by AP-2.

extensively than the synaptic vesicles (Fig. $9 D ; p<0.01$, Student's $t$ test). The two terminals injected with AP2 pep also had a larger plasma membrane area than the control terminals (Fig. 9E). This increase was attributable to the trapping of synaptic vesicle membrane in the plasma membrane, because the gain in plasma membrane area was very similar to the reduction in synaptic vesicle membrane area (Fig. 9E). The area of membrane in larger spherical structures, which have been termed vesicular endosomes (Burns et al., 1998), remained unchanged (Fig. 9E). Unlike disruptions of AP180 function alone (Zhang et al., 1998; Morgan et al., 1999; Nonet et al., 1999), the DLL-containing AP2 peptide did not alter synaptic vesicle size when it was injected into the presynaptic terminal (Fig. $9 F-H ; p<0.01$, Student's $t$ test). Apparently, simultaneously inhibiting both AP-2 and AP180 does not cause a change in vesicle size, consistent with previous notions that AP180 has a unique role in determining vesicle size (Ye and Lafer, 1995b;
Control

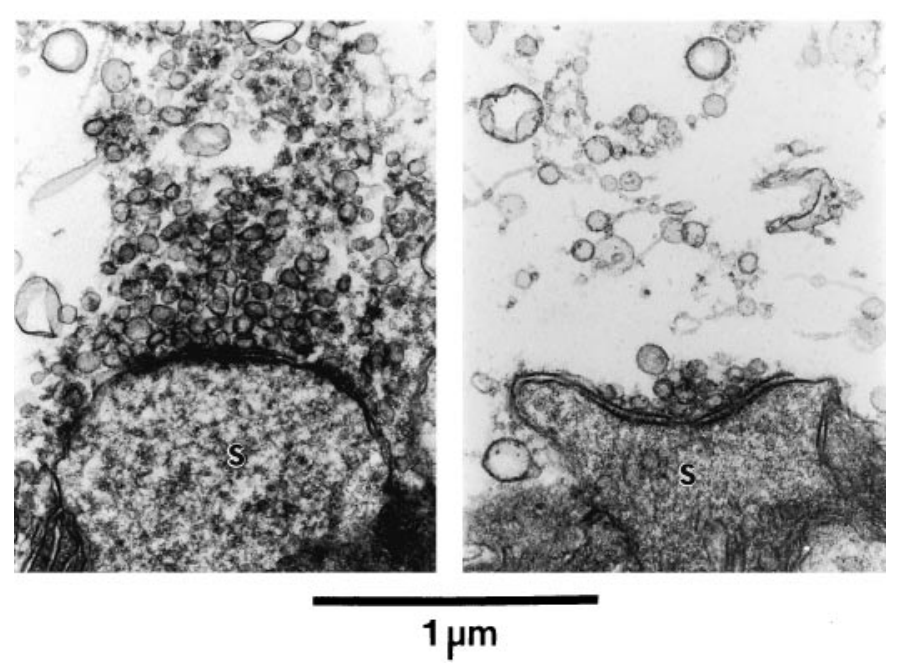

Figure 8. Presynaptic terminals injected with AP2 pep are depleted of synaptic vesicles. Terminals injected with AP2 pep had fewer synaptic vesicles than those injected with AP2 pep $\triangle \mathrm{DLL}$ (Control). The postsynaptic spines are indicated by $S$; the scale bar applies to both images.

Zhang et al., 1998; Morgan et al., 1999; Nonet et al., 1999). In summary, these results are consistent with AP2 pep blocking synaptic vesicle endocytosis at the clathrin assembly step and provide the final evidence that DLLs are functional clathrin assembly motifs.

\section{DISCUSSION}

We have identified a motif, containing a DLL or SLL sequence, that is found in multiple copies in many APs, including AP180, AP-1, AP-2, AP-3, and AP-4 (see Fig. 2). The conservation of these motifs suggests that they may have a general role in interactions with clathrin and could confer clathrin assembly properties to both tetrameric and monomeric APs. Our experiments provide several lines of evidence that this is the case. First, the clathrin assembly domain of AP180 (C58), which contains numerous DLL motifs, binds directly to the N-terminal domain of clathrin (see Fig. 1). Second, progressive deletion of the DLL motifs from AP180 gradually reduces the clathrin assembly activity of this protein in vitro (see Fig. 3). Third, peptides bearing DLL motifs from either AP180 (AP180 pep) or AP-2 (AP2 pep) cross-inhibit the ability of either full-length protein to promote clathrin assembly in vitro (see Figs. 4, 6, Table 1). Fourth, AP180 pep and AP2 pep reversibly inhibit synaptic transmission in vivo, presumably by blocking clathrin-mediated endocytosis (see Figs. 5, 7, Table 1). The effect of AP2 pep on clathrin assembly and synaptic transmission is attributable to the presence of the DLL motif, because mutating the DLL reduced these effects (see Table 1). For a series of mutant AP2 peptides the inhibition of synaptic transmission in vivo was correlated strongly with effects on clathrin assembly in vitro (see Figs. 6, 7, Table 1), suggesting that the inhibition of synaptic transmission is attributable to the inhibition of clathrin assembly in vivo. Finally, the DLL-containing AP2 pep produced structural changes consistent with the blockade of synaptic vesicle endocytosis at the clathrin assembly step; this peptide decreased the number of synaptic vesicles (see Figs. 8, 9) and expanded the presynaptic terminal plasma membrane area (see Fig. 9).

The AP180 and AP2 peptides used in this study have a low affinity for clathrin, reflected in the high concentrations required to inhibit both clathrin assembly in vitro and synaptic transmission in vivo. Despite their low affinity, our mutagenesis experiments indicate that these peptides bind to clathrin with high specificity (see Figs. 4, 6, Table 1). Thus, peptide affinity and specificity are independent. This conclusion is consistent with the finding that a peptide from the AP-2 $\beta$-subunit (GDLLNLDLGP), which is 
Figure 9. The DLL-containing AP2 pep inhibits synaptic vesicle endocytosis at the clathrin assembly step. $A$, Spatial distribution of synaptic vesicles $(S V s)$ in terminals injected with AP2 pep or controls. Data represent the mean values and SEM from 209 active zones $(A Z s)$ analyzed from two AP2 peptide-injected terminals and 184 AZs from two control terminals. $B$, Relative spatial distribution of SVs, determined by dividing the mean values for the AP2 pep by the control shown in $A$. The dashed line indicates a 1:1 ratio and represents no difference between terminals injected with AP2 pep or control peptide. $C, D$, AP2 pep significantly reduced the mean number of SVs $(C)$ and coated vesicles $(C V s ; D)$ found per AZ. $E$, The mean area of membrane within vesicular endosomes (Endo), SVs, CVs, and the presynaptic plasma membrane $(P M)$ was measured for each section in control and AP2 peptide-injected terminals. $F-H$, Distribution of SV diameters was measured from 20 active zones each in control $(F)$ or AP2 peptide-injected terminals $(G)$. The smooth lines indicate Gaussian functions fit to these distributions. $H$, Superimposition of the two distributions (normalized to the peak) reveals no change in mean SV diameter after injection of the AP2 peptide.
A

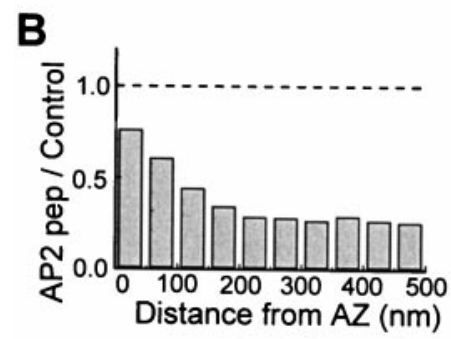

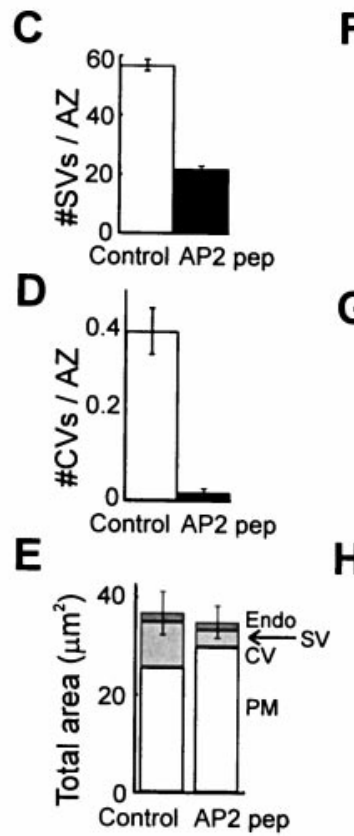
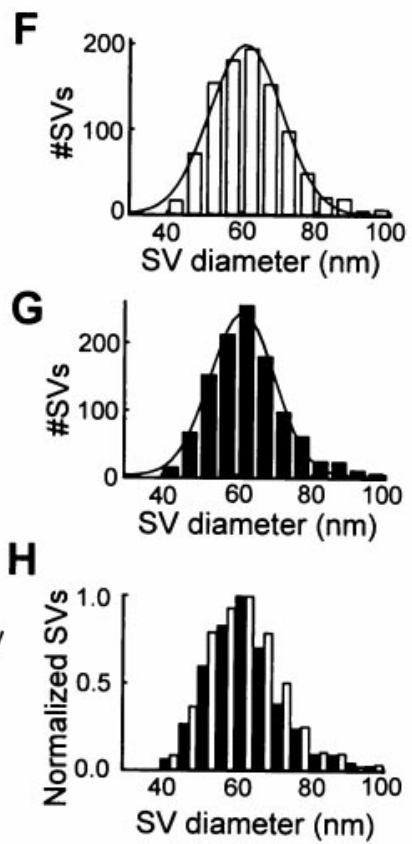

contained within our AP2 pep, specifically binds to the N-terminal domain of clathrin heavy chain (ter Haar et al., 2000) yet has a low binding affinity (T. Kirchhausen, personal communication).

It is unlikely that AP180 pep or AP2 pep prevented endocytosis by interfering with the functions of other AP binding partners, such as dynamin or amphiphysin. Although inhibiting these proteins causes accumulation of coated pits (Takei et al., 1996; Shupliakov et al., 1997), we never detected such structures in nerve terminals that were injected with these peptides. Instead, the structural changes produced by these peptides are consistent with the block of an earlier step in endocytosis, such as coated pit formation (Morgan et al., 1999). Thus, we conclude that DLL motifs within AP180 and AP-2 function as clathrin assembly motifs that are essential for the formation of clathrin coats during synaptic vesicle endocytosis. Given that AP-3 and AP-4 also contain similar sequences (see Fig. 2), it is likely that these APs also promote clathrin assembly and contribute to vesicular trafficking through other intracellular compartments.

The role of clathrin and its APs in synaptic vesicle recycling has been debated ever since Heuser and Reese (1973) first postulated that synaptic vesicles are recycled by a coated vesicle pathway. Recent observations of fast rates of endocytosis in some nerve terminals and secretory cells have led to suggestions of clathrinindependent endocytosis (Palfrey and Artalejo, 1998). Thus, the relative contribution of clathrin to synaptic vesicle recycling is not entirely clear. We found that presynaptic injection of a DLLcontaining peptide, which prevents clathrin assembly by both AP180 and AP-2, caused evoked neurotransmitter release to cease and dramatically reduced the number of synaptic vesicles and coated vesicles. Thus, our results indicate that clathrin assembly is an important step in the recycling mechanism even under low physiological rates $(0.03 \mathrm{~Hz})$ of neuronal activity, and complement several other studies in emphasizing the predominance of clathrinmediated endocytosis in nerve terminals (Gonzalez-Gaitan and Jackle, 1997; Zhang et al., 1998; Morgan et al., 1999; Ringstad et al., 1999). Whereas previous studies examined single components of clathrin assembly or later steps in clathrin-mediated endocytosis (Gonzalez-Gaitan and Jackle, 1997; Zhang et al., 1998; Morgan et al., 1999; Nonet et al., 1999; Ringstad et al., 1999), our experiments are the first to block in general all clathrin assembly in vivo by the clathrin APs.

The DLL motif that has been identified in this study bears a strong relationship to the recently described clathrin box sequence (consensus: LLpL-) that mediates the direct binding of AP-1, AP-2, AP-3, and $\beta$-arrestin to clathrin TD (Dell'Angelica et al., 1998; ter Haar et al., 2000). For example the DLL motif of AP2 pep is within a sequence (DLLNLD) that would be defined as a clathrin box. However, the DLL motif we have identified is somewhat more degenerate; in the case of AP180 pep the DLL motif is within a sequence (DLLGED) that is lacking the third L residue and intervening polar residue expected of a clathrin box. Despite this degeneracy, AP180 pep clearly interacts with clathrin, because it inhibits both AP180- and AP-2-mediated clathrin assembly in vitro (see Fig. 4) and synaptic transmission in vivo (see Fig. 5). In particular, the polar group that follows the di-leucine is not well conserved between the DLL motif and the clathrin box. However, available structural information indicates that this polar residue projects outward and lacks any specific interactions with clathrin (ter Haar et al., 2000). The DLL motif identified in this paper also bears some resemblance to a DXF motif hypothesized to mediate the binding of proteins to AP-2 (Owen et al., 1999). However, it is clear that the DLL motifs are not involved in AP-2 binding, because deletion of 10 of these motifs has no effect on the ability of AP180 to bind to AP-2 (Hao et al., 1999).

Our discovery that APs have multiple copies of the DLL motif is interesting in light of the structure of clathrin TD. TD has a seven-blade $\beta$-propeller structure with seven grooves between these blades (ter Haar et al., 1998). Clathrin box-containing peptides from AP-1, AP-2, AP-3, and $\beta$-arrestin- 2 bind to a groove between blades 1 and 2 of the TD, with the well conserved hydrophobic residues fitting into two hydrophobic pockets (ter Haar et al., 2000). Although there are no apparent repetitive features between the various grooves of TD, the hydrophobic nature of the grooves is well conserved (ter Haar et al., 1998). It is possible that the di-leucine pairs common to the DLL motif and clathrin box are involved in similar hydrophobic interactions with the grooves of TD, whereas the degeneracy of the DLL motifs may reflect differing groove specificities. Multivalent peptide-in-groove interactions have been observed in other $\beta$-propellers. For example, each groove of the five-blade tachylectin- $2 \beta$-propeller is an $N$-acetylglucosamine binding pocket (Beisel et al., 1999). Thus it is possible that APs have multiple clathrin binding motifs to allow for multiple interactions with the grooves between the blades of clathrin TDs.

The finding that individual APs contain multiple copies of the DLL motif also suggests that APs may promote clathrin assembly by cross-linking clathrin triskelia via multivalent interactions be- 


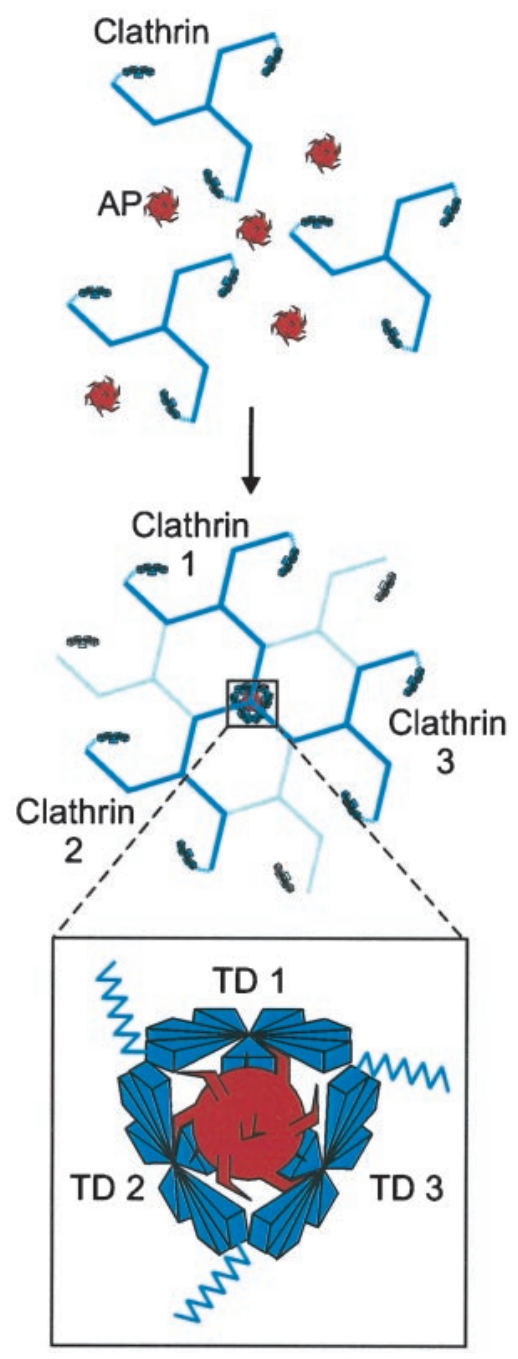

Figure 10. Cross-linking model for clathrin assembly by APs. Multiple clathrin binding elements (shown as spikes) exist within a single clathrin AP (top). These clathrin binding elements allow a single AP to have multivalent interactions with TDs from several clathrin molecules, thereby assembling them into a cage (middle). The inset (bottom) shows our proposed arrangement of TDs from three clathrin molecules at a vertex and illustrates the multivalent cross-linking of these clathrin molecules by APs.

tween repeated motifs in the APs and complementary binding sites in the clathrin TD (Fig. 10). In an assembled clathrin lattice three TDs from three different clathrin triskelia come together under each vertex (Smith et al., 1998; Musacchio et al., 1999). This places anywhere from 3-21 possible AP binding sites in close proximity, depending on whether only the groove between blades 1 and 2 contains an AP binding site or whether all seven grooves contain AP binding sites. AP180 and AP-2 form a complex that promotes clathrin assembly more efficiently than either protein alone (Hao et al., 1999), and each AP180/AP-2 complex contains up to 19 DLL motifs (see Fig. $2 A$ ). This leads us to hypothesize that the AP180/ AP-2 complex mediates clathrin assembly by cross-linking the TDs of three adjacent triskelia. These interactions could facilitate the movement of each triskelion into the lattice as it is assembling and assure an ordered size to the assembled structure on the basis of the geometric relationships between the proteins.

This model is supported by several observations. First, we show that the C-terminal domain of AP180, containing all twelve DLL motifs, binds directly to TD (see Fig. 1). Second, progressive deletion of the DLL motifs from AP180 gradually reduces the ability of AP180 to promote clathrin assembly, as though multiple sites on AP180 are binding to TD (see Fig. 3B). AP180 also contains more DLL motifs than are found in the sum of the $\alpha$ and $\beta$ subunits of AP-2 (see Fig. 2), which can explain why AP180 has a higher clathrin assembly activity in vitro than AP-2 (Lindner and Ungewickell, 1992). In our cross-linking model a given AP molecule interacts with TDs from three separate clathrin molecules (see Fig. 10). Because each clathrin molecule has three terminal domains, this could explain the observed stoichiometry of one AP180 per clathrin triskelion (Prasad and Lippoldt, 1988). Our model also may explain why $\beta$-arrestin does not promote clathrin assembly (Goodman et al., 1997); $\beta$-arrestin has only a single clathrin binding site, and our model would predict that this would prevent it from cross-linking adjacent triskelia (Goodman et al., 1997; Krupnick et al., 1997). One challenge to our model comes from the observation that clathrin was assembled weakly by an AP180 construct (C16) apparently containing only a single DLL motif (see Fig. 3). However, a DFL sequence at the C-terminal end of C16 (aa 899-901) may provide an additional clathrin binding site. This is consistent with a report that a similar motif within the final eight amino acids of yeast AP180A binds to clathrin (Wendland et al., 1999). Alternatively, it is possible that C16 contains still other clathrin binding sites that remain unidentified because of the degeneracy of the DLL motif. In either case the behavior of C16 then would be compatible with our model; although binding of an AP to three clathrin molecules may be optimal for efficient cross-linking, even binding to two molecules should be sufficient for some crosslinking to occur.

Until now, the basis for clathrin assembly by structurally diverse APs has been unclear. Our study indicates that this property is conferred by a conserved DLL motif that is found in multiple copies in all of these proteins. Further, our results suggest that this motif is part of a clathrin binding site that serves to assemble clathrin by acting as a multivalent cross-linker. These results provide novel insights into the molecular mechanism of clathrin assembly, a process that is essential for vesicle budding from donor membranes in presynaptic terminals and many other cellular compartments.

\section{REFERENCES}

Ahle S, Ungewickell E (1986) Purification and properties of a new clathrin assembly protein. EMBO J 5:3143-3149.

Ahle S, Mann A, Eichelsbacher U, Ungewickell E (1988) Structural relationships between clathrin assembly proteins from the Golgi and the plasma membrane. EMBO J 7:919-929.

Beisel HG, Kawabata S, Iwanga S, Huber R, Bode W (1999) Tachylectin-2: crystal structure of a specific GlcNAc/GalNAc-binding lectin involved in the innate immunity host defense of the Japanese horseshoe crab Tachypleus tridentatus. EMBO J 18:2313-2322.

Burns ME, Sasaki T, Takai Y, Augustine GJ (1998) Rabphilin-3A: a multifunctional regulator of synaptic vesicle traffic. J Gen Physiol 111:243-255.

Cowles CR, Odorizzi G, Payne GS, Emr SD (1997) The AP-3 adaptor complex is essential for cargo-selective transport to the yeast vacuole. Cell 91:109-118.

Dell'Angelica EC, Klumperman J, Stoorvogel W, Bonifacino JS (1998) Association of the AP-3 adaptor complex with clathrin. Science 280:431-434.

Dell'Angelica EC, Mullins C, Bonifacino JS (1999) AP-4, a novel protein complex related to clathrin adaptors. J Biol Chem 274:7278-7285.

Dreyling MH, Martinez-Climent JA, Zheng M, Mao J, Rowley JD, Bohlander SK (1996) The $\mathrm{t}(10 ; 11)(\mathrm{p} 13: \mathrm{q} 14)$ in the U937 cell line results in the fusion of the AF10 gene and CALM, encoding a new member of the AP-3 clathrin assembly protein family. Proc Natl Acad Sci USA 93:4804-4809.

Faundez V, Horng J-T, Kelly RB (1998) A function for the AP3 coat complex in synaptic vesicle formation from endosomes. Cell 93:423-432.

Goldstein JL, Brown MS, Anderson RG, Russell DW, Schneider WJ (1985) Receptor-mediated endocytosis: concepts emerging from the LDL receptor system. Annu Rev Cell Biol 1:1-39.

Gonzalez-Gaitan M, Jackle H (1997) Role of Drosophila $\alpha$-adaptin in presynaptic vesicle recycling. Cell 88:767-777.

Goodman Jr OB, Keen JH (1995) The $\alpha$ chain of the AP-2 adaptor is a clathrin-binding subunit. J Biol Chem 270:23768-23773.

Goodman Jr OB, Krupnick JG, Gurevich VV, Benovic JL, Keen JH (1997) Arrestin/clathrin interaction. Localization of the arrestin binding locus to the clathrin terminal domain. J Biol Chem 272:15017-15022.

Hao W, Luo Z, Zheng L, Prasad K, Lafer EM (1999) AP180 and AP-2 interact directly in a complex that cooperatively assembles clathrin. J Biol Chem 274:22785-22794. 
Hess SD, Doroshenko PA, Augustine GJ (1993) A functional role for GTP-binding proteins in synaptic vesicle cycling. Science 259:1169-1172.

Heuser JE, Reese TS (1973) Evidence for recycling of synaptic vesicle membrane during transmitter release at the frog neuromuscular junction. J Cell Biol 57:315-344.

Huang XQ, Miller W (1991) A time-efficient, linear-space local similarity algorithm. Adv Appl Math 12:337-357.

Keen JH (1987) Clathrin assembly proteins: affinity purification and a model for coat assembly. J Cell Biol 105:1989-1998.

Keen JH, Willingham MC, Pastan IH (1979) Clathrin-coated vesicles: isolation, dissociation, and factor-dependent reassociation of clathrin baskets. Cell 16:303-312.

Kirchhausen T (1999) Adaptors for clathrin-mediated traffic. Annu Rev Cell Dev Biol 15:705-732.

Kirchhausen T, Bonifacino JS, Riezman H (1997) Linking cargo to vesicle formation: receptor tail interactions with coat proteins. Curr Opin Cell Biol 9:488-495.

Kohtz DS, Puszkin S (1988) A neuronal protein (NP185) associated with clathrin-coated vesicles. Characterization of NP185 with monoclonal antibodies. J Biol Chem 263:7418-7425.

Krupnick JG, Goodman Jr OB, Keen JH, Benovic JL (1997) Arrestin/ clathrin: localization of the clathrin-binding domain of nonvisual arrestins to the carboxyl terminus. J Biol Chem 272:15011-15016.

Lindner R, Ungewickell E (1992) Clathrin-associated proteins of bovine brain coated vesicles. J Biol Chem 267:16567-16573.

Matsui W, Kirchhausen T (1990) Stabilization of clathrin coats by the core of the clathrin-associated protein complex AP-2. Biochemistry 29:10791-10798.

McMahon HT (1999) Endocytosis: an assembly protein for clathrin cages. Curr Biol 9:R322-R335.

Morgan JR, Zhao X, Womack M, Prasad K, Augustine GJ, Lafer EM (1999) A role for the clathrin assembly domain of AP180 in synaptic vesicle endocytosis. J Neurosci 19:10201-10212.

Murphy J, Keen JH (1992) Recognition sites for clathrin-associated proteins AP-2 and AP-3 on clathrin triskelia. J Biol Chem 267:10850-10855.

Murphy JE, Pleasure IT, Puszkin S, Prasad K, Keen JH (1991) Clathrin assembly protein AP-3. The identity of the $155 \mathrm{~K}$ protein, AP180, and NP185 and demonstration of a clathrin-binding domain. J Biol Chem 266:4401-4408.

Musacchio A, Smith CJ, Roseman AM, Harrison SC, Kirchhausen T, Pearse BMF (1999) Functional organization of clathrin in coats: combining electron cryomicroscopy and x-ray crystallography. Mol Cell 3:761-770.

Nonet ML, Holgado AM, Brewer F, Serpe CJ, Norbeck BA, Holleran J, Wei L, Hartwieg E, Jorgensen EM, Alfonso A (1999) UNC-11, a Caenorhabditis elegans AP180 homologue, regulates the size and protein composition of synaptic vesicles. Mol Biol Cell 10:2343-2360.

Owen DJ, Vallis Y, Noble MEM, Hunter JB, Dafforn TR, Evans PR, McMahon HT (1999) A structural explanation for the binding of multiple ligands by the $\alpha$-adaptin appendage domain. Cell 97:805-815.

Palfrey HC, Artalejo CR (1998) Vesicle recycling revisited: rapid endocytosis may be the first step. Neuroscience 83:969-989.

Pearse BM (1988) Receptors compete for adaptors found in plasma membrane coated pits. EMBO J 7:3331-3336.

Prasad K, Keen JH (1991) Interaction of assembly protein AP-2 and its isolated subunits with clathrin. Biochemistry 30:5590-5597.

Prasad K, Lippoldt RE (1988) Molecular characterization of the AP180 coated vesicle assembly protein. Biochemistry 27:6098-6104.

Ringstad N, Gad H, Low P, Di Paolo G, Brodin L, Shupliakov O, De
Camilli P (1999) Endophilin/SH3p4 is required for the transition from early to late stages in clathrin-mediated synaptic vesicle endocytosis. Neuron 24:143-154.

Robinson MS (1987) 100-kD coated vesicle proteins: molecular heterogeneity and intracellular distribution studied with monoclonal antibodies. J Cell Biol 104:887-895.

Sanchez ME, Nuno CM, Buchanan J, Augustine GJ (1990) Contractions of the squid stellate ganglion. J Exp Biol 152:369-387.

Schmid SL (1997) Clathrin-coated vesicle formation and protein sorting: an integrated process. Annu Rev Biochem 66:511-548.

Shih W, Gallusser A, Kirchhausen T (1995) A clathrin-binding site in the hinge of the $\beta 2$ chain of mammalian AP-2 complexes. J Biol Chem 270:31083-31090.

Shupliakov O, Low P, Grabs D, Gad H, Chen H, David C, Takei K, De Camilli P, Brodin L (1997) Synaptic vesicle endocytosis impaired by disruption of dynamin-SH3 domain interactions. Science 276:259-263.

Smith CJ, Grigorieff N, Pearse BM (1998) Clathrin coats at $21 \AA$ resolution: a cellular assembly designed to recycle multiple membrane receptors. EMBO J 17:4943-4953.

Sousa R, Tannery NH, Zhou S, Lafer EM (1992) Characterization of a novel synapse-specific protein. I. Developmental expression and cellular localization of the F1 protein mRNA. J Neurosci 12:2130-2143.

Takei K, Mundigl O, Daniell L, De Camilli P (1996) The synaptic vesicle cycle: a single vesicle budding step involving clathrin and dynamin. J Cell Biol 133:1237-1250.

Tebar F, Bohlander SK, Sorkin A (1999) Clathrin assembly lymphoid myeloid leukemia (CALM) protein: localization in endocytic-coated pits, interactions with clathrin, and the impact of overexpression of clathrinmediated traffic. Mol Biol Cell 10:2687-2702.

ter Haar E, Musacchio A, Harrison SC, Kirchhausen T (1998) Atomic structure of clathrin: a $\beta$ propeller terminal domain joins an $\alpha$ zigzag linker. Cell 95:563-573.

ter Haar E, Harrison SC, Kirchhausen T (2000) Peptide-in-groove interactions link target proteins to the $\beta$-propeller of clathrin. Proc Natl Acad Sci USA 97:1096-1100.

Thompson JD, Higgins DG, Gibson TJ (1994) CLUSTAL W: improving the sensitivity of progressive multiple sequence alignment through sequence weighting, position-specific gap penalties, and weight matrix choice. Nucleic Acids Res 22:4673-4680.

Wendland B, Steece KE, Emr SD (1999) Yeast epsins contain an essential $\mathrm{N}$-terminal ENTH domain, bind clathrin, and are required for endocytosis. EMBO J 18:4383-4393.

Ye W, Lafer EM (1995a) Clathrin binding and assembly activities of expressed domains of the synapse-specific clathrin assembly protein AP-3. J Biol Chem 270:10933-10939.

Ye W, Lafer EM (1995b) Bacterially expressed F1-20/AP-3 assembles clathrin into cages with a narrow size distribution: implications for the regulation of quantal size during neurotransmission. J Neurosci Res 41:15-26.

Zhang B, Koh YH, Beckstead RB, Budnik V, Ganetsky B, Bellen HJ (1998) Synaptic vesicle size and number are regulated by a clathrin adaptor protein required for endocytosis. Neuron 21:1465-1475.

Zhou S, Sousa R, Tannery NH, Lafer EM (1992) Characterization of a novel synapse-specific protein. II. cDNA cloning and sequence analysis of the F1-20 protein. J Neurosci 12:2144-2155.

Zhou S, Tannery NH, Yang J, Puszkin S, Lafer EM (1993) The synapsespecific phosphoprotein F1-20 is identical to the clathrin assembly protein AP-3. J Biol Chem 268:12655-12662. 